\title{
Use of clinical guidelines: perspectives from clinicians in paediatric and maternity hospitals in Kabul, Afghanistan
}

\author{
H. Graham, ${ }^{1}$ M. Tokhi, ${ }^{2}$ A. Edward,${ }^{3}$ A.S. Salehi, ${ }^{4}$ S. Turkmani, ${ }^{5}$ T. Duke ${ }^{2}$ and L. Bartlett ${ }^{3}$
}

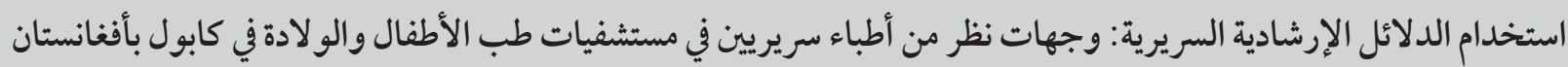

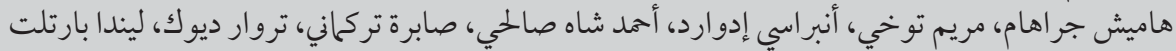

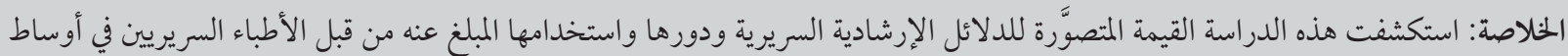

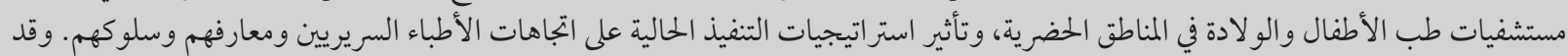

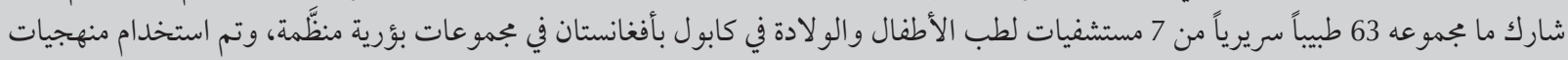

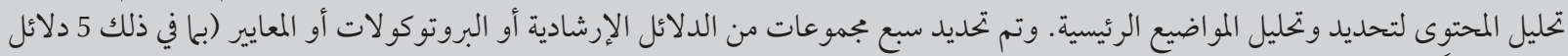

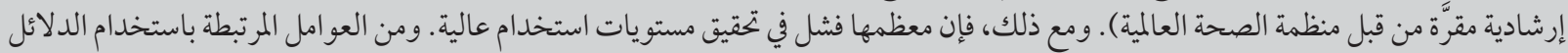

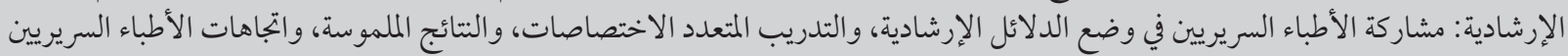

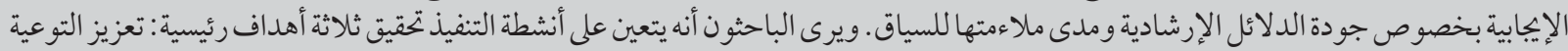

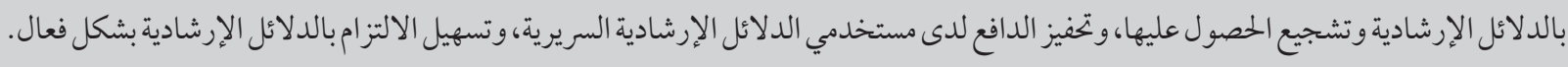

ABSTRACT This study explored the perceived value, role and reported use of clinical guidelines by clinicians in urban paediatric and maternity hospital settings, and the effect of current implementation strategies on clinician attitudes, knowledge and behaviour. A total of 63 clinicians from 7 paediatric and maternity hospitals in Kabul, Afghanistan participated in structured focus groups; content analysis methodology was used for identification and analysis of key themes. Seven sets of guidelines, protocols or standards were identified (including $5 \mathrm{WHO}$-endorsed guidelines). However, most are failing to achieve high levels of use. Factors associated with guideline use included: clinician involvement in guideline development; multidisciplinary training; demonstrable results; and positive clinician perceptions regarding guideline quality and contextual appropriateness. Implementation activities should fulfil 3 major objectives: promote guideline awareness and access; stimulate motivation among clinical guideline users; and actively facilitate adherence to guidelines.

Application des lignes directrices cliniques : points de vue des cliniciens dans des hôpitaux pédiatriques et des maternités à Kaboul (Afghanistan)

RÉSUMÉ La présente étude a analysé l'application des lignes directrices cliniques ainsi que la valeur et le rôle qui leur sont attribués par des cliniciens exerçant dans des hôpitaux pédiatriques et des maternités en milieu urbain. L'effet des stratégies de mise en œuvre actuelles sur les attitudes, les comportements et les connaissances des cliniciens a également été étudié. Au total, 63 cliniciens exerçant dans sept hôpitaux pédiatriques et maternités de Kaboul (Afghanistan) ont participé à des groupes de discussion thématique structurés. Une méthodologie d'analyse de contenu a été utilisée pour identifier et analyser les thèmes clés. Sept ensembles de lignes directrices, protocoles ou normes ont été identifiés (y compris cinq lignes directrices approuvées par l'OMS). Toutefois, la plupart de ceuxci n'atteignent pas de hauts niveaux d'application. Les facteurs associés à l'application des lignes directrices sont les suivants : I'implication du clinicien dans l'élaboration des lignes directrices; une formation pluridisciplinaire ; des résultats démontrables; des perceptions cliniques positives de la qualité des lignes directrices, mais aussi leur adéquation par rapport au contexte. Les activités de mise en œuvre devraient atteindre les trois principaux objectifs suivants : faire mieux connaître les lignes directrices et promouvoir leur accès ; accentuer la motivation des utilisateurs des lignes directrices cliniques; et faciliter activement le respect des lignes directrices.

'Department of General Paediatrics, Royal Children's Hospital, Melbourne, Australia. ${ }^{2}$ Centre for International Health, Burnet Institute, Melbourne, Australia (Correspondence to M. Tokhi: mariam.tokhi@burnet.edu.au). ${ }^{3}$ Department of International Health, Johns Hopkins School of Public Health, Baltimore, United States of America. ${ }^{4}$ Health Financing Directorate, Ministry of Public Health, Kabul, Afghanistan. ${ }^{5}$ Afghan Midwifery Association, Islamic Republic of Afghanistan; Centre for Midwifery, Child and Family Health, University of Technology, Sydney, Australia.

Received: 12/05/14; accepted: 04/12/14 


\section{Introduction}

Clinical guidelines are systematically developed statements that seek to distil and present the best available evidence in a clear and practical way for clinicians and the community (1). The World Health Organization (WHO) has embraced guideline development as one of its core functions, recognizing their important role in translating evidence into practice and thereby improving health outcomes (2). This is particularly well appreciated in maternal and child health where guidelines are seen as instrumental in filling the implementation gap between evidence of quality, cost-effective health interventions and their full implementation $(3,4)$. However, despite strong evidence of their potential benefits many guidelines fail, either because of deficits in the quality of their evidence base or due to failures in implementation $(5,6)$. And while there is emerging consensus regarding the development of quality guidelines, there is significant debate over the best implementation strategies (7-11).

Evidence from a variety of settings suggest that clinical guideline implementation can be impeded by barriers or aided by facilitators at the level of the guideline itself, the individual patient and clinician, the work environment and the broader organizational and structural environment (12). However, specific factors vary greatly between guidelines and settings, underlining the importance of a context-specific approach to guideline development and implementation $(7,13)$.

\section{Clinical guidelines in Afghanistan}

Questions regarding how to best implement clinical guidelines are particularly relevant in low-resource settings where resource allocation for implementation strategies is most limited $(14,15)$. Afghanistan has invested substantially in guideline development over the past decade, while facing the challenges of war and resource scarcity. Afghanistan ranks 175 th (out of 185 countries) on human development index (HDI) rankings, with one of the highest child and maternal mortality rates globally (under-5 mortality 64 per 1000 live births; maternal mortality ratio 327 per 100000 live births) (16). Health workforce capacity is at one-third of WHO recommendations (7.26 doctors/nurses/midwives per 100000 population) $(17,18)$ and poor quality of care is impairing progress in maternal and child health $(19,20)$. Professional and pharmaceutical regulation is weak, research capacity and access to evidence is severely limited, and large gaps remain in health service management, broader social support systems and infrastructure (17).

Within this context, the presence of clinical guidelines has been shown to be a major predictor of clinical care and overall hospital performance, with hospitals in Kabul identified as particularly deficient $(18,21,22)$. Multiple international partners have been involved in guideline development and the Ministry of Public Health (MoPH) has made "enhancing evidence-based decision making" one of its 10 strategic objectives and begun creation of a clinical guidelines unit $(23,24)$.

This is an exploratory study into guideline use in Afghan paediatric and maternity hospitals and addresses: which clinical guidelines are reported to be available; how these guidelines are perceived and used by clinicians; what factors facilitate or impede implementation of these guidelines; and what can be learned from these data about clinical guideline implementation in other lowresource settings.

\section{Methods}

\section{Study design}

A qualitative study design was chosen, using focus group discussions to collect data and a content analysis approach to objectively identify key themes. This was accompanied by site visits to record objective evidence of guideline use and supplementary meetings with relevant $\mathrm{MoPH}$ officials (directors of child health, maternal health and quality improvement sections). The study protocol was developed in consultation with clinicians in Afghanistan and structured around 4 themes: clinical decision-making resources; guideline availability and use; guideline value and role; and factors influencing guideline implementation. To encourage free and open discussion we chose peer interaction among clinician groups (e.g. trainee paediatric doctors), and for consistency the same 2 investigators (H.G., M.T.) moderated all focus groups. The research was approved by the institutional review boards of Johns Hopkins University and the MoPH Afghanistan.

\section{Participants and recruitment}

Hospitals were identified in collaboration with the $\mathrm{MoPH}$ and selected to represent a spectrum of urban obstetric and paediatric hospitals, including those which are regarded as particularly influential through their clinical, academic and health policy activities. These included Afghanistan's 2 largest paediatric referral and teaching hospitals, 2 largest obstetric referral and teaching hospitals, 2 district-level urban hospitals and 1 private hospital. Participants were selected purposively through contact with hospital directors to represent clinician groups with different levels of experience. To facilitate participant comfort and openness focus groups were conducted in a private area of the participants' hospital (with one additional focus group of midwives conducted at the Afghan Midwifery Association). No monetary incentive was offered for participation. Focus groups were conducted in English with Dari translation. 


\section{Data collection}

Written consent was obtained from all participants. The interview guide contained open-ended questions and prompts and was designed to address major implementation factors identified in previous systematic reviews $(12,14)$ and conceptual frameworks $(7,25)$. It was pilot-tested on junior clinicians from non-English speaking backgrounds in the United States of America and Afghanistan. All sessions were audio-recorded and accompanied by written field notes, with independent confirmatory translation as necessary.

\section{Data analysis}

Transcription, indexing and coding of data was conducted by the same investigators who led the focus groups to maintain consistency. After verification of accuracy, a content analysis approach was used to individually analyse each transcript, code the data and categorize ideas into broad themes. The results were then reviewed by both investigators (H.G., M.T.) to identify findings that were applicable to the entire study, with reference back to the transcripts to confirm or refute emerging hypotheses.

Guideline use was evaluated using Pathman's awareness to adherence framework (25), with scores of low, medium and high corresponding to whether each criterion (aware/agree/ adopt/adhere $)$ pertained to few $(<1$ in 3), some (1-2 in 3), or most (> 2 in 3) clinicians. This was correlated with objective evidence of guideline use during site visits. Comparison tables were constructed to assess whether there was any association between guideline use and any of the implementation factors identified from focus groups.

\section{Results}

A total of 22 focus group discussions were conducted in January 2013 and involved 63 clinicians, including 43 doctors and 20 nurses/midwives
(Table 1). Senior doctors (paediatricians and obstetricians) generally did not have formal qualifications (due to limited opportunity for postgraduate specialty training in Afghanistan until recently), but all had greater than 5 years of experience and were working in supervisory and teaching roles. All trainees were undertaking postgraduate training in paediatrics or obstetrics, and had between 1 and 4 years of clinical experience. Midwives had formal midwifery training and varied in the number of years of clinical experience (from 1 year to $>5$ years). Each focus group involved up to 6 clinicians and was approximately 45-60 minutes in duration. Gender distribution reflected the Afghan norm of predominantly female doctors and midwives in maternity hospitals and male doctors and nurses in other hospitals.

\section{Guideline availability}

In total 7 sets of guidelines, protocols or standards were identified in Kabul paediatric and maternity hospitals. Five of these were WHO guidelines: Managing Complications in Pregnancy and Childbirth (IMPAC) (26); Integrated Management of Childhood Illness (IMCI) (27); Pocketbook of Hospital Care for Children in Low-Resource Settings (Pocketbook) (28); Emergency Triage, Assessment and Treatment (ETAT) (29); and Training Course on the Management of Severe Malnutrition
(Malnutrition guideline) (30). The remaining 2 included the MoPH Hospital Standards Manual (MoPH Standards), and the CURE International Hospital (a private hospital department of obstetrics and gynaecology) Protocol Handbook (CURE Handbook). All were available in the national Dari language, except for the CURE Handbook which was only in English (IMCI was also available in Pashto).

Of these, the IMCI, Pocketbook and IMPAC guidelines were all widely known. However, IMPAC was the only guideline that reached moderate or high levels of use in all relevant study hospitals. The ETAT, Malnutrition and CURE guidelines were not widely available but reached moderate or high levels of use in individual hospitals. Table 2 summarizes the main findings regarding guideline availability, use and implementation strategies.

\section{Key themes: role, value and factors associated with use of guidelines}

Key themes from the focus groups are listed in Table 3 with selected quotations for illustration.

Clinical guidelines have a small role in clinical practice in Kabul paediatric hospitals and their role as decision-making aids is poorly appreciated. Despite a general belief that "if guidelines were available they would be used", deeper

\begin{tabular}{lcccc}
\hline \multicolumn{3}{l}{ Table 1 Characteristics of the study participants } & & \\
\hline Profession & Males & Females & Total \\
& No. & No. & No. & $\%$ \\
Paediatrician & 8 & - & 8 & 14 \\
Paediatric trainee & 8 & - & 8 & 18 \\
Nurse (paediatric) & 5 & 1 & 6 & 10 \\
Obstetrician & 1 & 9 & 10 & 18 \\
Obstetric trainee & - & 17 & 17 & 31 \\
Midwife (hospital) & - & 7 & 7 & 13 \\
Midwife (AMA) & - & 7 & 7 & 13 \\
Total & 22 & 41 & 63 & 100 \\
\hline
\end{tabular}

AMA = Afghan Midwifery Association (hosted 1 additional focus group with midwives from a variety of urban and rural hospitals). 


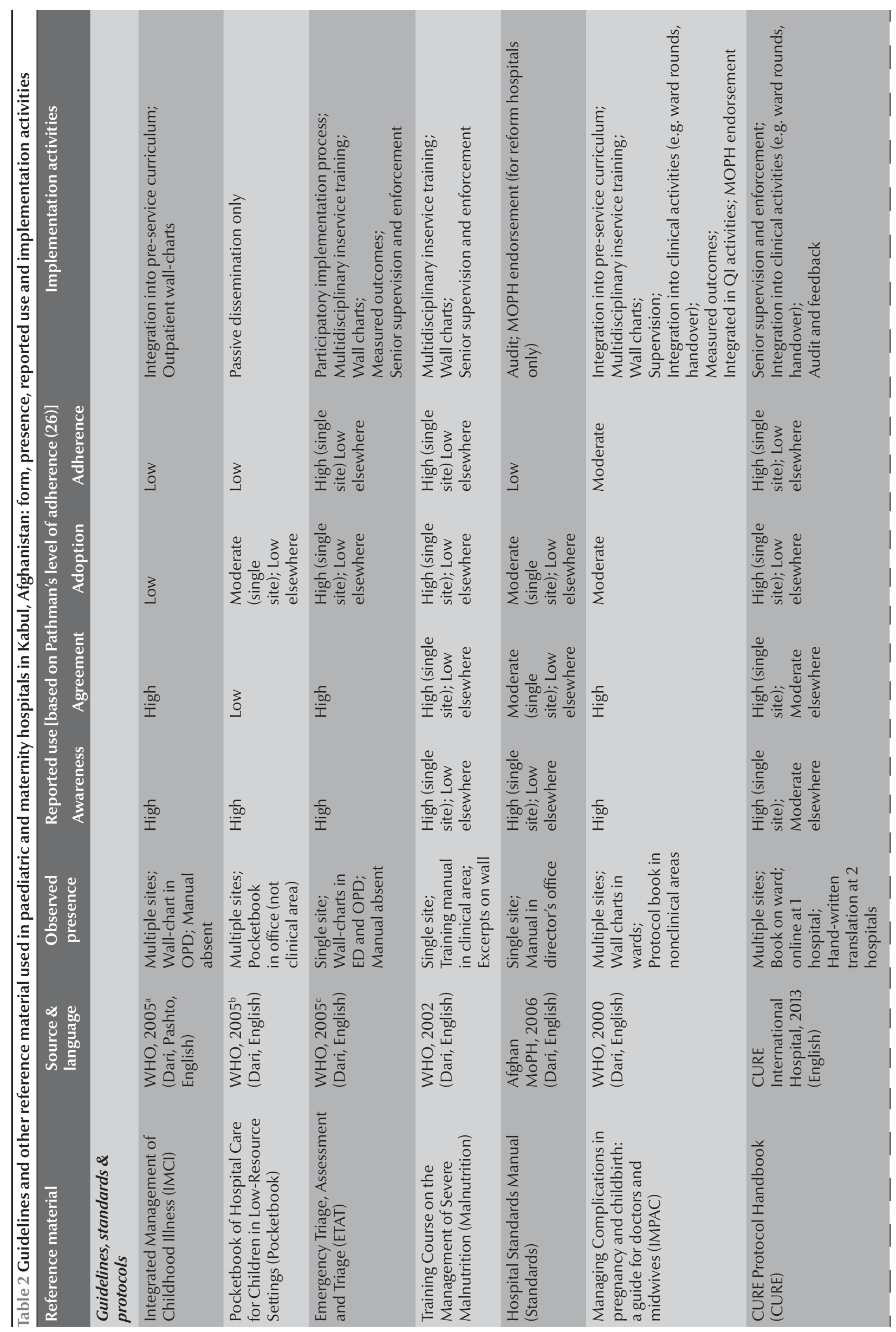


exploration revealed many factors that influenced the use of guidelines (Box 1).

While reportedly highly valued, guidelines are rarely consulted within clinical settings. There is an expectation that doctors should not need assistance with decision-making and that "it should already be in their head". Consulting resources on the job could mean that "other people will think they are illiterate about their profession". Participants reported that the most frequent use of guidelines on the job was when they were available as wall charts.

Guidelines are most often identified as educational resources, with minimal distinction from textbooks, websites and training materials. Printed guidelines are commonly viewed as "simple textbooks" that are superseded by more comprehensive textbooks. Guidelines that were viewed exclusively as educational references had lower reported levels of use.

Guidelines are valued for helping to define and enhance the clinical role of nurses and midwives: both by doctors and by nurses and midwives themselves. As such, guidelines have challenged the assumption that only doctors make clinical decisions and have promoted effective task-shifting. Guidelines that effectively delineated clinical roles had higher levels of adoption and use.

Nurses and midwives reported valuing guidelines, and particularly guideline-related training opportunities, for broadening their employment prospects and enhancing their professional respect. However, this motivation to participate in guideline-related training activities was not associated with increased use.

Clinicians described the value of guidelines in setting a context-specific standard and resolving conflict between different resources. Guidelines such as IMPAC and the Malnutrition guideline were valued for being more appropriate to the health needs, cultural context and service provision capacities of Afghanistan than other existing resources (such as academic textbooks). Guidelines which clinicians viewed as contextually appropriate had higher levels of reported adoption.

Clinicians reported valuing guidelines which delivered tangible results, particularly improvements in patient outcomes and ease of clinical duties. Evidence of these benefits was reported to be one of the most compelling reasons for clinicians to use guidelines. While many participants reported subjective evidence of effect, guidelines that had activities that sought to measure and report outcomes (e.g. audit and feedback) had higher levels of reported use.

Midwives and doctors were interested in how guidelines were developed (or adapted); describing that there should be a participatory approach. Those who had been involved in guideline development described it as generating ownership of guidelines among hospital staff and motivating clinicians to invest in their implementation. Midwives described disappointment 


\section{Role and value}

Doctors should know what to do

\section{Educational resource}

Define and enhance clinical roles of midwives and nurses

\section{Context-appropriate standard}

Clarification of conflict

\section{Factors influencing use}

Deliver tangible results / Audit and feedback

Clinician involvement in

Perceived as up-to-date

Integrated \& multi-disciplinary training

Systemic challenges in the work environment

Ministry of Public Health endorsement

Influence of senior doctors guideline development

"If a doctor (looks at) a book in front of a patient the patient will think he is (simple), he doesn't know anything... This is a big problem here in Afghanistan."

"[The Pocketbook] is good... but it does not have as many details as in the other textbooks." "Lots of improvements have happened between doctors and midwives because now midwives are receiving essential obstetric care [IMPAC] courses... and they know about the guidelines... Doctors trust them now... they know that they can do everything for the patient and now there is more respect between doctors and midwives. "

"The ETAT program enabled nurses to be able to evaluate a patient... and do the right things for the patient... Before, the nurse cannot evaluate, or distinguish, what this patient needs."

"When I go to the last edition of Nelsons, the 19th edition, I see only 5 or 6 sentences about mumps. But here I have many patients with mumps... The diseases which are common in Afghanistan, they are a lot different to the ones in the US or in Europe. That is one of the important reasons we should have a specific guideline to follow that."

"CURE guidelines advise IV infusion of magnesium sulfate [for severe pre-eclampsia]. But, we don't have instruments for a pump infusion to regulate magnesium sulfate infusion for patients with severe pre-eclampsia or eclampsia. We just do it with a simple IV line... and with difficulty we adjust the drops. There is not an IV pump... or even enough stands to hang the IV infusions or staff to follow the infusion."

"Right now, the problem is that we don't have one universal guideline that we can implement... The problem here is that we ask the trainees to use the CURE protocol for hypertensive patients. But then the trainer comes along and says: 'OK I don't use that CURE protocol. I don't like that hospital. I have my own...'. So we cannot enforce it."

"This ETAT programme, the aim is to reduce the mortality rate in the first 24 hours... At the beginning the (proportion of deaths occurring in the first 24 hours) was $57-58 \%$, it was high... After 6 months from implementing this programme, we reduced it to 33\%."

"All of the doctors and specialists in the paediatric department they should sit together and make a guideline for all the standard cases..."

"We should be making the standards here (in Afghanistan) and we should make a decision on the basis on the pathology of Afghanistan."

"There is no update in the [WHO Pocketbook]. I think it is about 6, 8 years ago... But here we have very poor and very ill patients. We need to use the updated guidelines. [Therefore we refer] mostly to textbooks... like Nelsons."

"We [doctors] take the ETAT training for 3 days... the person who is sitting at the gate, he must be trained about the ETAT. Also the nurse. So the patient can reach very soon the place for examination."

"I graduated by reading the IMPAC book so it is easy. We are reading the IMPAC book in midwifery school, then we are coming to practicals and our teacher is teaching us according to that, and then we are practicing according to that."

"There is no quality check in our country... When we use a multinational drug from [company $\mathrm{X}$, we don't know if it is the original drug or not... We see the packet. It is beautiful packet, so we think the drug is beautiful. So we use the drug from the package."

"At other hospitals we had lack of instruments, lack of facilities, lack of knowledge of the patient, lack of time... We had lots of limitations for applying [maternity] guidelines in the government hospitals... but here the limitations are very little. The administration is very supportive-they find out what we need. They are very cooperative with us."

"There are some doctors here that didn't take the ETAT programme, because they are junior, but the senior doctors have talked, have explained about the ETAT programme and they are doing accordingly."

"[The Pocketbook] is not a national guideline. It is just a [resource] from WHO and the Child and Adolescent Department of the Ministry of Public Health... The Malnutrition guideline it is a national guideline, it is used all over Afghanistan, if you are in Kabul, if you are in Mazar, if you are in Jalalabad, all of you will use this national guideline for malnutrition."

IMPAC = Integrated Management of Pregnancy and Childbirth; ETAT = Emergency Triage Assessment and Treatment; CURE = CURE Protocol Handbook; Pocketbook = Pocketbook of Hospital Care for Children in Low-Resource Settings; Nelson Textbook of Pediatrics 19th edition; Training Course on the Management of Severe Malnutrition (Malnutrition). 


\section{Box 1 Factors that influenced use of guidelines in Afghan paediatric and maternity hospitals}

\section{Level of the guideline}

Epidemiologically and contextually appropriate content

Presence of conflict between available guidelines

Lack of distinction between clinical guidelines and textbooks

Integration of guidelines into clinician training

Availability as job aids (e.g. wall charts)

Availability in local languages

\section{Level of individual clinicians (and patients)}

Belief that referring to reference material shows lack of clinician competence Perception that clinical guidelines exist only for junior doctors and midwives Quality of interdisciplinary relations (e.g. doctor-midwife relationship)

Perception that guidelines are up-to-date and evidence-based

Perception that guidelines are appropriate to the local context

\section{Level of clinical work environment}

Magnitude of workload and patient demand

Adequacy of facilities and equipment to follow guidelines

Human resource management (e.g. adequate staffing and safe hours)

Multidisciplinary involvement in guideline use

Supervision and enforcement of guideline use by senior clinicians

\section{Level of broader organizational and structural milieu}

Clinician involvement and trust in guideline development

Coordination and endorsement by Ministry of Public Health

Strength and quality of pre-service and professional education

Supportive pre-service (medical, nursing and midwifery) supervision

Emphasis on medical ethics during clinician training (e.g. patient-centred care)

with the lack of midwifery representation during guideline development; although nurses did not expect to be involved. Clinicians emphasized the need for multidisciplinary, multihospital representation when developing guidelines. Guidelines that had strong clinician participation in their development were more likely to be accepted by clinicians.

Clinicians reported that guidelines should be available in local languages (Dari and Pashto) as well as English (particularly for nurses and midwives who have less English language training). Translation into local languages was a necessary but not sufficient factor for guideline adoption; a number of guidelines that have been translated were not widely used.

Clinicians reportedly value guidelines that are up-to-date, context-specific, comprehensive and evidence-based. Yet clinicians' subjective perceptions of these qualities do not always correlate with objective assessments of currently available resources. For example, the Pocketbook was critiqued for being out-of-date and overly simplistic, while ETAT was described as up-to-date, and appropriately detailed (yet, both the Pocketbook and ETAT guidelines were published in 2005 and ETAT's contents are fully contained within the Pocketbook). Guidelines perceived as up-to-date, comprehensive and evidence-based had higher levels of reported acceptance and use.

Training was reported to be a key strategy for overcoming resistance from clinicians to adopting guidelines. While any training was valued by clinicians, only training that was multidisciplinary, practice-based and multimethod correlated positively with guideline acceptance and use. Furthermore, a multidisciplinary approach was reported to enhance team dynamics and professional communication between doctors and nurses/midwives. Guidelines that were integrated into medical, midwifery and nursing curricula had higher levels of reported awareness and acceptance (though not necessarily adherence).

Many barriers to guideline use were reported in the work environment, including: inadequate staffing; poor drug quality and supply; inadequate facilities; and lack of equipment and poor maintenance. Poor resource management and communication between clinicians and administrators reportedly compounded these deficiencies.

Senior doctors' opinions reportedly carried immense weight and influenced junior doctors' perception of and reported adherence to clinical guidelines. 


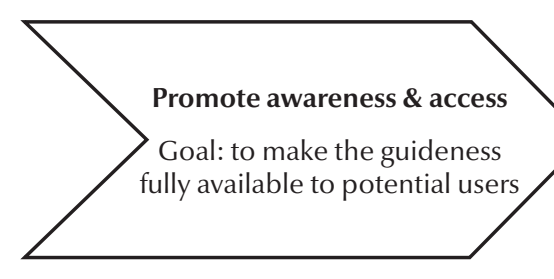

- Translation into local language (s)

- Dissemination of printed material

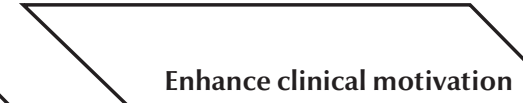 \\ Goal: to persuade clinicians of the benefits of guidelines \\ - Multidisciplinary clinician involvement in guideline development and implementation \\ - generate local consensus building among opinion leaders \\ - optimise contextual appropriateness and acceptability to clinician-users}

- Multidisciplinary training for specific guidelines

- demonstrate the unique of guidelines compared to other educational materials (i.e. what guidelines are, who they are for, and how to use them)

- demonstrate guideline(s) to be up-to-date and authoratative

- demonstrate guideline(s) are epidemiologically appropriate

- Integration in the curriculum

- Role delineation for doctors and nurses/midwives

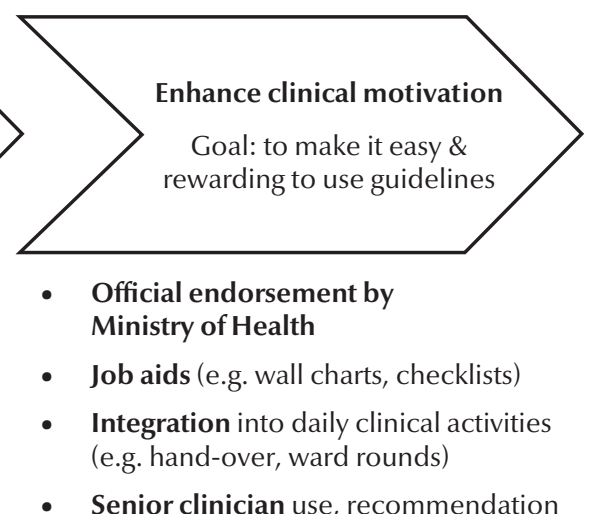

- Senior clinician use, recommendation and enforcement of guidelines

- Measurement of results and feedback to clinicians

- demonstrate positive effects from using guidelines (e.g. improved patient outcomes, easing of clinical workload)

- Supportive management systems

- responsive to clinician needs (e.g. rostering, workload, equipment supply, infrastructure)

- promoting a culture of learning and accountability

Figure 1 Framework for implementation of guidelines: 3 key tasks, illustrated with examples from Kabul paediatric and maternity hospitals

MoPH support and endorsement of clinical guidelines was highly valued by Afghan clinicians. Guidelines that carried such endorsement had higher levels of reported adoption.

\section{Discussion}

Clinical guidelines are recognized as having an important role in Afghan hospitals; however, most are failing to achieve high levels of use despite demand from users and commitment from administrators (24). This paper proposes a framework for enabling successful guideline implementation in Afghanistan, and describes 5 core principles of guideline implementation. While this exploratory study limited its focus to urban hospitals in Afghanistan, these principles may be relevant to other low and middle-income countries facing similar challenges.
It should be noted that this study was limited to clinicians based at major paediatric and maternity hospitals in urban Kabul and it evaluated guideline use at a single point in time. This enabled us to compare the use of guidelines at different stages of implementation and across a range of institutions (including the largest paediatric and maternity hospitals). However, this approach cannot fully capture the dynamic nature of clinician behaviour over time, and therefore extrapolation of the findings to other settings (especially non-urban settings) may be limited. Assessing guideline use based on clinicians' reports (rather than direct observation) enabled the collection of qualitative data on why clinicians use guidelines, but risked introducing reporting bias. To increase the validity of this we combined use of Pathman's adherence framework, corroboration across participants and objective observation of the presence of guidelines.

\section{Framework for effective guideline implementation}

Dissemination of clinical guidelines alone is ineffective in changing clinician practice $(14,15)$. We propose that effective implementation strategies must achieve 3 stepwise goals. First, implementation strategies must promote awareness of and access to guidelines, making them fully available to potential users. Secondly, they must enhance motivation among guideline users, persuading them of the benefits of using guidelines. Thirdly, they must actively facilitate adherence to guidelines, making it rewarding for clinicians to use them. Figure 1 illustrates this framework with examples from Afghan hospitals.

Conceptually, this may explain why there have been only modest 
improvements in clinical care from single interventions (e.g. dissemination of educational material; audit and feedback; reminders and "job aids"; patientdirected initiatives; opinion leaders; and financial incentives), and conflicting results when such interventions are combined (12,31). Interventions should be conceived and combined to address each step of the framework sequentially (access, motivation, adherence) and no single intervention can be expected to fulfil them all.

\section{Core principles for effective guideline implementation}

This study highlights a number of principles that are core to successful guideline implementation. First, clinician-users must be held at the centre of guideline development and implementation. Although the importance of consumer involvement in the guideline development process has been well documented, it is frequently neglected (12,31-33). This may be particularly true in post-conflict and resource-constrained settings in which the task of guideline development and implementation often falls on nongovernmental organizations and other agencies (and coordinated advocacy by professional groups is weak). Our findings suggest that involving clinicians can increase acceptance and appreciation of guidelines by clinicians, build consensus among opinion leaders and empower clinicians to optimize the clinical work environment. Furthermore, it offers the opportunity to use local knowledge and experience to optimize the contextual appropriateness of guidelines: a critical but commonly neglected activity.

Secondly, there is need for a multidisciplinary approach to guidelines. Involvement of both doctors and nurses/midwives with guidelines in Afghan hospitals was not only strongly associated with their acceptance and use, but was also reported to deliver benefits that went far beyond the scope of the guidelines themselves. These benefits included improved role delineation, task shifting and enhanced recognition and respect for midwives and nurses: all of which are much needed in Afghanistan (34). This finding is particularly interesting given the enormous impact that these professional relationships have on quality of care, worker satisfaction and patient outcomes, and the paucity of effective interventions to improve these relationships (35).

Training is commonly recognized as having a critical role in implementing clinical guidelines, although there is great variation in the types of training and how effective it is in changing clinician behaviour $(12,14,15)$. Characteristics of effective guideline training include: small groups; focussed topics; multiple teaching methods (e.g. role play, practising skills); informed by local culture and context; and explicitly addressing barriers and facilitators to guideline use $(14,15)$. Our findings suggest that training can be one of the most effective motivators for clinicians to use guidelines, particularly if it is able to address key concerns of clinicians. For Afghan clinicians this included explicitly demonstrating the guideline(s) to be up-to-date and authoritative, and epidemiologically and contextually appropriate. While these are all essential characteristics of a good guideline, whether a guideline is perceived as such is more critical in determining whether a clinician will use it than whether it is objectively so. It is also important to address the unique role and utility of guidelines as compared to other resource and educational materials (i.e. what guidelines are, who they are for, and how to use them). If a written guideline is not distinguished from other written educational resources it will always risk being seen as too simple or lacking authority. Similarly, if training on guidelines does not go beyond skills training to explicitly address how to use the guideline, it is likely to get lost amidst other educational activities and not be translated into guideline use.

Our study revealed higher use of guidelines in institutions where quality improvement activities were established. Quality improvement activities are based on the plan-do-study-act cycle and typically include standardbased audit and feedback, the creation of job aids (e.g. wall charts, checklists), supportive supervision and attention to improving clinical processes. Given that clinicians reported proof of effectiveness as one of the strongest motivators for them to use guidelines, institutionalizing a measurement and feedback process may improve and sustain the use of guidelines (so long as this produces results). Additionally, health-care systems failures were reported as major barriers to guideline use (and quality of care in general) and quality improvement activities provided a way for clinicians to engage in addressing them. Thus, our study supports previous findings that implementing guidelines within a broader quality improvement process is more effective and sustainable $(15,31)$.

Finally, the MoPH and professional organizations have an important role in supporting the implementation of guidelines. Formal endorsement of guidelines was a strong determinant of their use in Afghan hospitals, and clinicians were eager to have endorsed national standards. This may provide an opportunity to facilitate guideline development and implementation activities nationally, provide legitimacy to guidelines and related implementation activities, and use credentialing to foster institutional environments that are conducive to guideline implementation.

\section{Conclusions}

This exploratory study into the use of paediatric and maternity clinical 
guidelines suggests that guidelines are wanted by clinicians, and can be effectively implemented despite Afghanistan's challenging current context. However, attention must be directed towards implementation activities that: promote awareness and access; enhance clinician motivation; and facilitate clinician adherence to guidelines.

Particular interventions to achieve this are identified and may be useful to inform guideline activities in Afghanistan and other low-resource settings. Further studies to develop this guideline implementation framework and to explore the comparative effectiveness and utility of various implementation strategies are needed in other contexts.

\section{Acknowledgements}

We gratefully acknowledge the contributions of: Dr Alishah Alawi, Dr Younusi Motawali, Dr Najiba Yaftali, Dr Karima Mayar Amiri and Dr Yousuf Rahimi (MOPH Afghanistan); Dr Nasrat (Jhpiego Afghanistan);
Dr Paul Ickx, Dr Zafar Omari and Abdul Zahir Siddiqui (MSH Afghanistan); Dr Mirwas Rahimzai, Dr Rita Hashemi Saalem and Dr Mirwais Amiri (URC Afghanistan); Dr Hameed Elmyar, Dr Hazeeb Rehman and other hospital clinicians; Dr Jim Heiby and Dr Rashad Massoud (USAID).

Funding: This study was partially supported by the Johns Hopkins Bloomberg School of Public Health Travel award.

Competing interests: None declared.

\section{References}

1. Field MJ, Lohr KN. Clinical practice guidelines: directions for a new program. Washington (DC): National Academies Press; 1990.

2. WHO guidelines approved by the Guidelines Review Committee. Geneva: World Health Organization; 2006 (http:// www.who.int/publications/guidelines/en/, accessed 4 January 2015).

3. Haines A, Kuruvilla S, Borchert M. Bridging the implementation gap between knowledge and action for health. Bull World Health Organ. 2004 Oct;82(10):724-31, discussion 732. PMID:15643791

4. Chopra M, Sharkey A, Dalmiya N, Anthony D, Binkin N; UNICEF Equity in Child Survival, Health and Nutrition Analysis Team. Strategies to improve health coverage and narrow the equity gap in child survival, health, and nutrition. Lancet. 2012 Oct 13;380(9850):1331-40. PMID:22999430

5. Grimshaw JM, Russell IT. Achieving health gain through clinical guidelines II: Ensuring guidelines change medical practice. Qual Health Care. 1994 Mar;3(1):45-52. PMID:10136260

6. Kryworuchko J, Stacey D, Bai N, Graham ID. Twelve years of clinical practice guideline development, dissemination and evaluation in Canada (1994 to 2005). Implement Sci. 2009;4:49. PMID:19656384

7. Gagliardi AR, Brouwers MC, Palda VA, Lemieux-Charles L, Grimshaw JM. How can we improve guideline use? A conceptual framework of implementability. Implement Sci. 2011;6:26. PMID:21426574

8. Fretheim A, Schünemann HJ, Oxman AD. Improving the use of research evidence in guideline development: 15 . Disseminating and implementing guidelines. Health Res Policy Syst. 2006;4:27. PMID:17156459

9. Turner T, Misso M, Harris C, Green S. Development of evidence-based clinical practice guidelines (CPGs): comparing approaches. Implement Sci. 2008;3:45. PMID:18954465

10. English M, Opiyo N. Getting to grips with GRADE-perspective from a low-income setting. J Clin Epidemiol. 2011 Jul;64(7):70810. PMID:21316192

11. Brouwers M, Kho M, Browman G, Burgers J, Cluzeau F, Feder G, et al. AGREE II: Advancing guideline development, reporting and evaluation in healthcare. CMAJ. 2010 Dec 14;182(18):E83942. PMID:20603348

12. Francke AL, Smit MC, de Veer AJE, Mistiaen P. Factors influencing the implementation of clinical guidelines for health care professionals: a systematic meta-review. BMC Med Inform Decis Mak. 2008;8:38. PMID:18789150

13. Harrison MB, Légaré F, Graham ID, Fervers B. Adapting clinical practice guidelines to local context and assessing barriers to their use. CMAJ. 2010 Feb 9;182(2):E78-84. PMID:19969563

14. Siddiqi K, Newell J, Robinson M. Getting evidence into practice: what works in developing countries? Int J Qual Health Care. 2005 Oct; 17(5):447-54. PMID:15872024

15. Rowe AK, de Savigny D, Lanata CF, Victora CG. How can we achieve and maintain high-quality performance of health workers in low-resource settings? Lancet. 2005 Sep 1723;366(9490):1026-35. PMID:16168785

16. Afghanistan Mortality Survey 2010. Calverton (MD): ICF Macro; 2011 (http://www.usaid.gov/sites/default/files/documents/1871/Mortality\%20Survey\%202010.pdf, accessed 4 January 2015).

17. Afghanistan National Health Workforce Plan 2012-16. Kabul: General Directorate of Human Resources; 2012. (http://www. who.int/workforcealliance/countries/Afghanistan_HRHplan_2012_draft.pdf, accessed 4 January 2015).

18. Edward A, Kumar B, Niayesh H, Naeem AJ, Burnham G, Peters $\mathrm{DH}$. The association of health workforce capacity and quality of pediatric care in Afghanistan. Int J Qual Health Care. 2012 Dec;24(6):578-86. PMID:23081907

19. Lind A, Edward A, Bonhoure P, Mustafa L, Hansen P, Burnham G, et al. Quality of outpatient hospital care for children under 5 years in Afghanistan. Int J Qual Health Care. 2011 Apr;23(2):108-16. PMID:21242157

20. Kim Y-M. Partamin, Ansari N, Khalid Y. Emergency obstetric and neonatal care (EmONC) needs assessment in Afghanistan. Kabul: United National Children's Fund; 2010.

21. Edward A, Dwivedi V, Mustafa L, Hansen PM, Peters DH, Burnham $G$. Trends in the quality of health care for children aged less than 5 years in Afghanistan, 2004-2006. Bull World Health Organ. 2009 Dec;87(12):940-9. PMID:20454485

22. The balanced scorecard report for Afghanistan hospitals 2011/12. Kabul: Ministry of Public Health Afghanistan; 2012.

23. National strategy for improving quality in healthcare: $2011-2015$ (1390-1394). Kabul: Ministry of Public Health Afghanistan; 2011.

24. Rahimi YA. Clinical guideline development in Afghanistan: conceptual framework. Kabul: Ministry of Public Health Afghanistan; 2012. 
25. Pathman DE, Konrad TR, Freed GL, Freeman VA, Koch GG. The awareness-to-adherence model of the steps to clinical guideline compliance. The case of pediatric vaccine recommendations. Med Care. 1996 Sep;34(9):873-89. PMID:8792778

26. Mathai M, Sanghvi H, Guidotti RJ, Broekhuizen F, Johnson R, Foster-rosales A, et al. Integrated Management of Pregnancy and Childbirth (IMPAC). Managing complications in pregnancy and childbirth: a guide for midwives and doctors. Geneva: World Health Organization; 2000.

27. Integrated Management of Childhood Illness (IMCI) [Internet] Geneva: World Health Organization; 2005 (http://www.who. int/maternal_child_adolescent/topics/child/imci/en/, accessed 4 January 2015).

28. Pocket book of hospital care for children. Guidelines for the management of common illnesses with limited resources. 1st ed. Geneva: World Health Organization; 2005 (http://whqlibdoc.who.int/publications/2005/9241546700.pdf?ua=1, accessed 4 January 2015).

29. Emergency Triage Assessment and Treatment (ETAT). Manual for participants. Geneva: World Health Organization; 2005 (http://whqlibdoc.who.int/publications/2005/9241546875 eng.pdf, accessed 4 January 2015).

30. Training course on the management of severe malnutrition. Geneva: World Health Organization; 2002 (http://www.who. int/nutrition/publications/severemalnutrition/training_inpatient_MSM/en/, accessed 4 January 2015).

31. Grimshaw JM, Thomas RE, MacLennan G, Fraser C, Ramsay CR, Vale $\mathrm{L}$, et al. Effectiveness and efficiency of guideline dissemination and implementation strategies. Health Technol Assess. 2004 Feb;8(6):iii-iv, 1-72. PMID:14960256

32. Boivin A, Currie K, Fervers B, Gracia J, James M, Marshall C, et al.; G-I-N PUBLIC. Patient and public involvement in clinical guidelines: international experiences and future perspectives. Qual Saf Health Care. 2010 Oct;19(5):e22. PMID:20427302

33. Fretheim A, Schünemann HJ, Oxman AD. Improving the use of research evidence in guideline development: 5 . Group processes. Health Res Policy Syst. 2006;4:17. PMID:17140442

34. Bartlett L, Manalai P, Azfar P, Callaghan R, Turkmani S, Assefi $\mathrm{N}$, et al. Evaluation of the pre-service midwifery education program in Afghanistan. Kabul: Ministry of Public Health Afghanistan; 2011.

35. Zwarenstein M, Goldman J, Reeves S Interprofessional collaboration. Interprofessional collaboration: effects of practicebased interventions on professional practice and healthcare outcomes. Cochrane Database Syst Rev. 2009; (3):CD000072. PMID:19588316 